\title{
Upconversion Fluorescence-SERS Dual-Mode Tags for Cellular and in Vivo Imaging
}

\author{
Xiaojuan Niu, ${ }^{\dagger \dagger}$ Haiyan Chen, ${ }^{\dagger, \S}$ Yunqing Wang, ${ }^{*, \perp}$ Wenhai Wang, ${ }^{\perp}$ Xiuyan Sun, ${ }^{\ddagger}$ and Lingxin Chen ${ }^{*, \perp}$ \\ ${ }^{\ddagger}$ School of Pharmacy, Yantai University, Yantai 264005, China \\ ${ }^{\S}$ School of Life Science and Technology, State Key Laboratory of Natural Medicines, China Pharmaceutical University, Nanjing, \\ 210009, China \\ ${ }^{\perp}$ Key Laboratory of Coastal Environmental Processes and Ecological Remediation, Yantai Institute of Coastal Zone Research, Chinese \\ Academy of Sciences, Yantai 264003, China
}

\section{Supporting Information}

ABSTRACT: Fluorescent-surface enhanced Raman scattering (F-SERS) dual mode tags showed great potential for bioimaging due to the combined advantages of intuitive, fast imaging of fluorescence and multiplex capability of SERS technique. In previously reported F-SERS tags, organic fluorescent dyes or quantum dots were generally selected to generate fluorescence signal. Herein, we reported the first proof-of-concept upconversion fluorescence (UCF)-SERS dual mode tags based on near infrared (NIR) laser $(980 \mathrm{~nm}$ ) excited upconversion nanoparticles (UCNPs) for live-cell and in vivo imaging. Three components involved in this tag: $\mathrm{NaYF}_{4}: \mathrm{Yb}$,Er UCNPs@SiO ${ }_{2}$ serving as the fluorescent core of the tag; silver nanoparticles in situ grown on the surface of

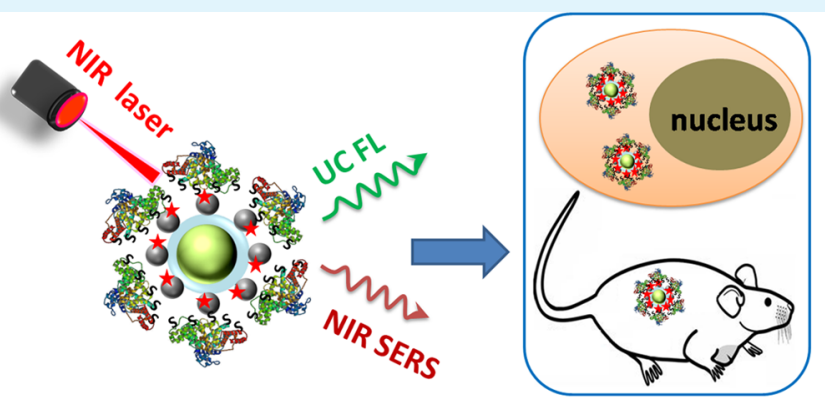

Upconversion FL-SERS tag UCNPs@SiO 2 for generating characteristic Raman signal; and denatured BSA coating rendering the tag's stability and biocompatibility. The UCF-SERS tags integrated the NIR imaging capability of both fluorescent UCNPs and plasmonic SERS nanoprobe, which facilitated dual mode bioimaging investigation, especially for living animals. Ex vivo experiments revealed that with $980 \mathrm{~nm}$ and $785 \mathrm{~nm}$ NIR laser irradiations, the UCF and SERS signals of the tags could be detected from 3 and $7 \mathrm{~mm}$ deep pork tissues, respectively. Furthermore, the in vivo imaging capabilities of UCF-SERS tags were successfully demonstrated on living mice. The developed dual modality tags held great potential for medical diagnostics and therapy.

KEYWORDS: upconversion nanoparticles, silver nanoparticles, fluorescence, surface-enhanced Raman scattering (SERS),

dual-mode imaging

\section{INTRODUCTION}

Surface-enhanced Raman scattering (SERS) tag was a kind of novel light scattering optical probe composed by noble metal nanoparticles (NPs), Raman reporter molecules and surface protection materials. ${ }^{1}$ SERS tag possessed many advantages over fluorescence dyes and quantum dots (QDs) for their tremendous multiplexing capacity, quantification capability, high photostability and biocompatibility. Up to now, they had been successfully applied for molecular detection, ${ }^{2-4}$ live-cell sensing, ${ }^{5,6}$ tissues diagnosis ${ }^{7}$ and in vivo imaging. ${ }^{8}$

To extend the functionality of SERS tags, recently several SERS-related multimodal probes had been developed, such as tags integrating SERS with fluorescence, ${ }^{9-11} \mathrm{X}$-ray computed tomography (CT), ${ }^{12}$ or magnetic resonance imaging (MRI) signals. ${ }^{13,14}$ Among them fluorescent-SERS (F-SERS) dual mode tags were the most extensively investigated. As is wellknown, SERS was superior from the aspect of multiplex capability, whereas it was inferior in imaging speed. By contrast, fluorescence imaging was intuitive and faster than SERS imaging, but its multiplex sensing ability was not satisfactory, especially for in vivo investigation. The application of F-SERS dual mode tags was promising to improve the imaging quality: the fluorescence severed as a fast indicator and the SERS signal was used to distinguish specific targets in multiplex interactions. Recently, F-SERS tags have been successfully used for the duplex detection of different markers co-expressed on breast cancer cells ${ }^{10}$ and in vivo fluorescence imaging, SERS detection, and photodynamic therapy in living mouse. ${ }^{15,16}$

The selection of fluorophores determined the fluorescent properties of F-SERS tags. Organic dyes derivatives, such as fluorescein isothiocyanate (FITC), Rhodamine B isothiocyanate (RBITC)-labeled silica precursors, ${ }^{9,10,17}$ or polyelectrolytes $^{18,19}$ had been selected to generate fluorescence of dual mode tags via the conjugation on the SERS cores through

Received: January 20, 2014

Accepted: March 11, 2014

Published: March 11, 2014 
Scheme 1. Synthesis of UCF-SERS Dots and the Imaging Mode
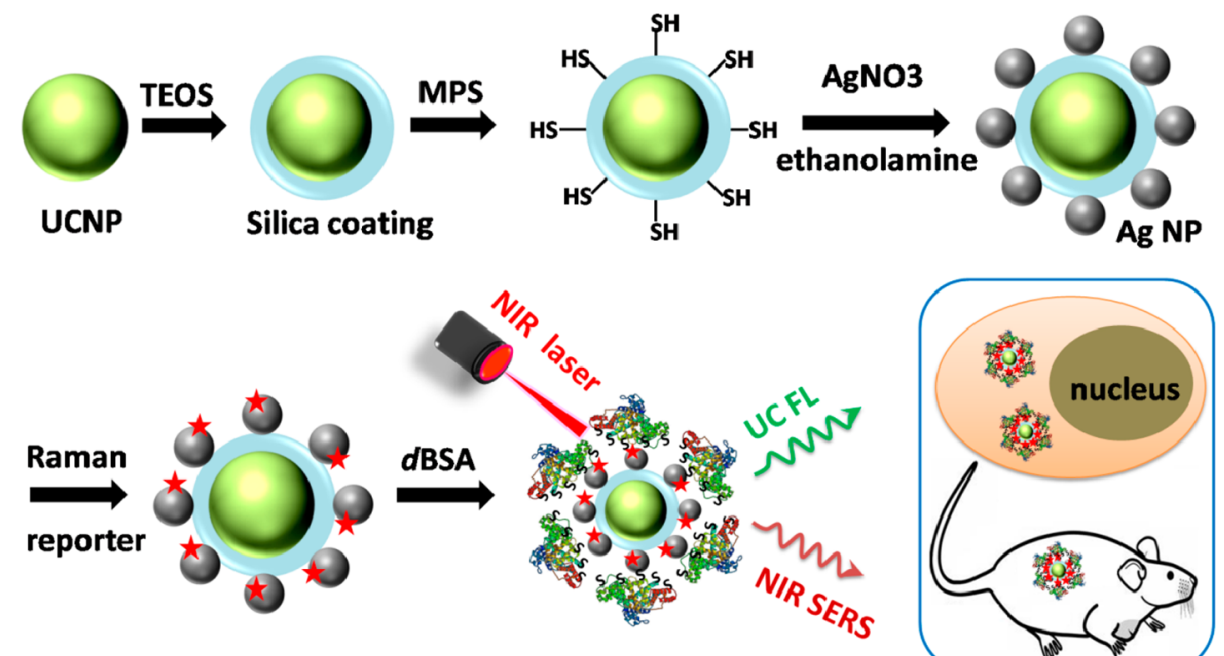

FL-SERS tag

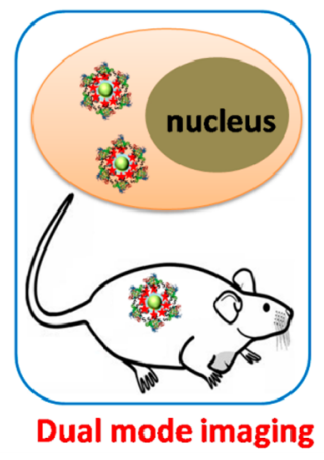

silane chemistry or electrostatic force induced layer-by-layer deposition. RBITC was also reported to directly adsorb onto $\mathrm{Ag},{ }^{20}$ acting as both Raman reporter and fluorescence generator. Recently, Cui's group carried out serial works on design and application of F-SERS tags by using QDs as fluorescence emitters. $^{21,22}$ Compared with organic dyes, QDs were superior because of the narrow emission peaks and multiple colors. By separately modifying two distinct SERS tags with different colored QDs, interesting multiple codes in a permutation combination manner could be obtained, which further enlarge the multiple labeling ability of sole QDs or SERS tags. ${ }^{23}$

In recent years, lanthanide-based upconversion nanoparticles (UCNPs) had drawn great interests in biological application owing to their unique fluorescence mechanism, which absorbed $980 \mathrm{~nm}$ near infrared (NIR) light (a biologically transparent window), and emit high energy visible photons through an upconversion process. ${ }^{24}$ UCNPs shared similar advantages with QDs such as narrow emission bandwidth, tunable multicolor, while overcame some limitations such as photostability, photoblinking and cytotoxicity. ${ }^{25-27}$ These advantages made UCNPs ideal candidates for developing multifunctional theranostic nanoplatforms combining optical imaging, drug delivery, and photodynamic therapy that used at the level of living cells and small animals. ${ }^{28-32}$

We speculated that UCNPs and plasmonic noble metal NPs were to be good fit to produce upconversion fluorescence (UCF)-SERS probe owing to the following two reasons. (1) NIR excitation of UCNPs had a deep penetration depth in tissue, making UCNPs suitable for in vivo applications. Meanwhile, NIR SERS tags were also reported for small animal imaging. The combination of these two NIR compatible probes might generate novel dual mode tags that could deliver more valuable in vivo information. (2) Recent published works revealed that the assembling of UCNPs with gold ${ }^{33}$ and silver $\mathrm{NPs}^{34}$ or shells ${ }^{35}$ (typical noble metal NP SERS substrates) could enhance the UCNPs' luminescence by one order of magnitude at optimal conditions. This unique up-regulating plasmonic modulation effect was crucial to obtain satisfactory fluorescence sensitivity during the construction of F-SERS tags. By contrast, when using organic dyes or QDs as fluorophores, endeavors should be made to preserve the fluorescence because it was easily quenched by adjacent noble metal NPs.

To prove our speculations, we performed the first proof-ofprinciple investigation on dual modality probes combining UCF and SERS signals for living cell and small animal imaging. As shown in Scheme 1, the UCF-SERS tag was composed of three components. The first was silica coated $\mathrm{NaYF}_{4}: \mathrm{Yb}, \mathrm{Er}$ UCNP, which served as the fluorescent core of the tag. The second was a typical SERS-tag structure building from Ag NPs in situ grown on silica layer and different kinds of Raman reporters. Furthermore, denatured BSA ( $d \mathrm{BSA}$ ) was coated on the tag to improve the colloidal stability and biocompatibility. The UCF and SERS properties of the as-prepared tags were deeply studied and the bioimaging capabilities were demonstrated on MCF-7 cells and a living mouse.

\section{EXPERIMENTAL SECTION}

Materials. $\mathrm{RE}_{2} \mathrm{O}_{3}(\mathrm{RE}=\mathrm{Y}, \mathrm{Yb}$, and Er), oleic acid (OA, 90\%), 1octadecen (ODE, 90\%), ammonium fluoride and 3-(4,5-dimethylthiazol-2-yl)-2,5-diphenyltetrazolium bromide (MTT) were purchased from Aladdin Reagent Company. CO-520 (average molecular weight of 441), 3-mercaptopropyl-triethoxysilane (MPS, 80\%), 3, 3'diethylthiadicarbocyanine iodide (DTDC), 3, 3'-diethylthiatricarbocyanine iodide (DTTC), bovine serum albumin (BSA) were purchased from Sigma-Aldrich. Tetraethylorthosilicate (TEOS), sodium hydroxide, sodium borohydride, silver nitrate, ethylene glycol, ethanolamine, crystal violet (CV), malachite green (MG) and rhodamine 6G (Rh6G) were purchased from China National Medicine Corporation Ltd. All chemicals were used as received without further purification. $\mathrm{RECl}_{3}$ were prepared by dissolving the corresponding $\mathrm{RE}_{2} \mathrm{O}_{3}$ in hydrochloric acid solution. The products were evaporated and redissolved in distilled water.

Characterization. The transmission electron microscopy (TEM) images were acquired on a JEM-1400 transmission electron microscope (JEOL, Japan). UV/vis/NIR absorption spectra were recorded on a Thermo Scientific NanoDrop 2000/2000C spectrophotometer. The hydrodynamic diameters were measured on a Zetasizer Nano ZS90 (Malvern, UK). The UCF spectra were measured on a FluoroMax-4 spectrofluorometer (HORIBA, France) by using a 980 $\mathrm{nm}$ laser $(1.0 \mathrm{~W})$ as the excitation source. SERS spectra acquired from $632.8 \mathrm{~nm}$ laser irradiation were recorded on a DXR Raman Microscope (Thermal Fisher, USA) with a laser power of $2 \mathrm{~mW}$. The sample solutions were filled in glass capillary with a diameter of $0.1 \mathrm{~mm}$ and focused by a $10 \times$ microscope objective. SERS spectra 


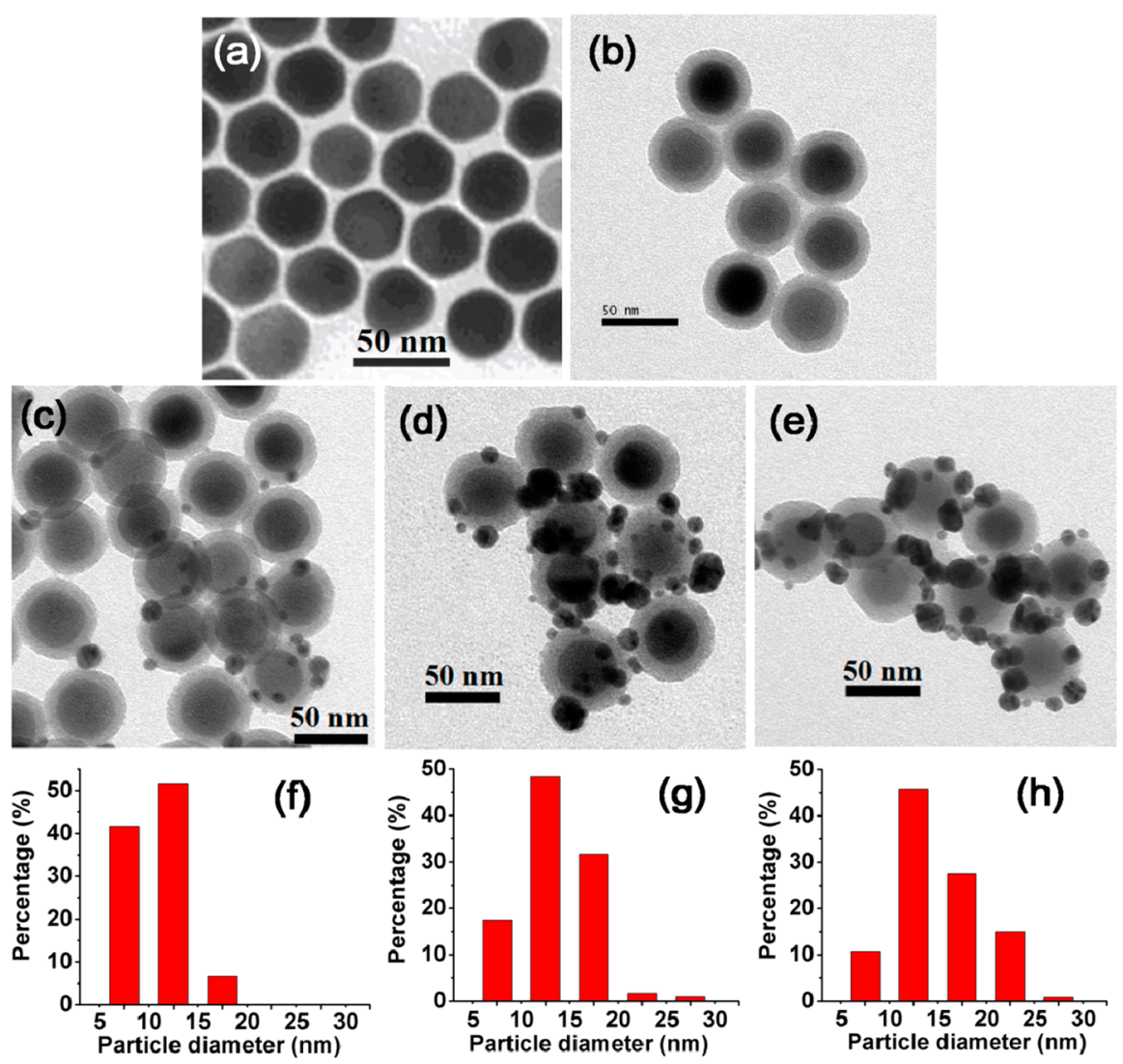

Figure 1. TEM images of (a) $\mathrm{NaYF}_{4}: \mathrm{Yb}$,Er UCNPs, (b) UCNP@SiO $\mathrm{NPs}_{2}$ (c-e) showed TEM images of UCNP@SiO $\mathrm{U}_{2} @ \mathrm{Ag}$ nanocomposites synthesized with 1,2 , and $3 \mathrm{mM} \mathrm{AgNO}_{3 .} ;(\mathrm{f}-\mathrm{h})$ showed the corresponding Ag NP size distribution histograms obtained by analyzing $120 \mathrm{Ag}$ NPs of the three UCNP@SiO ${ }_{2} @ \mathrm{Ag}$ nanocomposite samples.

obtained from $785 \mathrm{~nm}$ laser irradiation were detected using an Agility Raman spectrometer (BaySpec, USA). The laser power was $200 \mathrm{~mW}$ and the integration time was $1 \mathrm{~s}$. The testing solutions were dropped on a silicon wafer before SERS measurements.

Synthesis of $\mathrm{NaYF}_{4}: \mathrm{Yb}, \mathrm{Er}$ UCNPs. $\mathrm{NaYF}_{4}: \mathrm{Yb}, \mathrm{Er}$ UCNPs were synthesized following a published work. ${ }^{36}$ Briefly, $\mathrm{RECl}_{3}(1 \mathrm{mM}$, $\mathrm{Y}: \mathrm{Yb}: \mathrm{Er}=78: 20: 2)$ were added to a three-necked flask containing $\mathrm{OA}$ $(6 \mathrm{~mL})$ and $\mathrm{ODE}(15 \mathrm{~mL})$. The mixture was heated to $160^{\circ} \mathrm{C}$ for 30 min and then cooled down to room temperature. Afterwards, $10 \mathrm{~mL}$ methanol solution of $\mathrm{NaOH}(2.5 \mathrm{mM})$ and $\mathrm{NH}_{4} \mathrm{~F}(4 \mathrm{mM})$ was added slowly and the solution was stirred for another $30 \mathrm{~min}$. After evaporation of methanol, the mixture was rapidly heated to $300{ }^{\circ} \mathrm{C}$ under stirring in nitrogen atmosphere for $1 \mathrm{~h}$ and then cooled to room temperature. The resulting NPs were precipitated by the addition of ethanol, separated by centrifugation, washed several times with ethanol, and redispersed in cyclohexane.

Synthesis of UCNP@SiO 2 NPs. One-half of a milliliter of CO-520, $8 \mathrm{~mL}$ of cyclohexane, and $2 \mathrm{~mL}$ of UCNP solution were mixed and stirred for $10 \mathrm{~min}$. One-tenth of a milliliter of $30 \mathrm{wt} \%$ ammonia was then added and the container was sealed and sonicated for $20 \mathrm{~min}$ until a transparent emulsion was formed. Eight-hundreths of a millimeter of TEOS was then added into the solution. The solution was stirred for $48 \mathrm{~h}$ at a speed of $600 \mathrm{rpm}$. UCNP@SiO $\mathrm{SPs}_{2}$ were precipitated by adding acetone, washed with ethanol/water $(1: 1 \mathrm{v} / \mathrm{v})$ twice, and then redispersed in $6 \mathrm{~mL}$ of ethanol.

Synthesis of UCNP@SiO ${ }_{2} @ A g$ Nanocomposites. One-quarter of a milliliter of UCNP@SiO ${ }_{2} \mathrm{NPs}$ was added into $4.5 \mathrm{~mL}$ of $1.5 \% \mathrm{w} /$ $\mathrm{w}$ MPS in ethanol, and stirred for $20 \mathrm{~h}$ at $25{ }^{\circ} \mathrm{C}$. The product was centrifuged and washed with ethanol twice to remove the excess MPS. The thiol-modified UCN@SiO ${ }_{2} \mathrm{NPs}$ were equally separated into four $1.5 \mathrm{~mL}$ eppendorf tubes. Three of the samples were redispersed in 0.8 $\mathrm{mL}$ of $1.0,2.0$, and $3.0 \mathrm{mM} \mathrm{AgNO}_{3}$ (ethylene glycol) solution, followed by adding $0.08 \mathrm{~mL}$ of $100 \mathrm{mM}$ ethanolamine (ethylene glycol) solution after $30 \mathrm{~min}$. These three tubes were placed on a shaker for $12 \mathrm{~h}$ at $25^{\circ} \mathrm{C}$. The obtained NPs were centrifuged and washed with ethanol twice, and redispersed in $0.8 \mathrm{~mL}$ of water.

Synthesis of dBSA-Capped UCF-SERS Tag. $0.5 \mathrm{~mL}$ of asprepared UCNP@SiO ${ }_{2} @ \mathrm{Ag}$ nanocomposite solution was mixed with $0.1 \mathrm{~mL}$ of different Raman reporter solutions (CV, MG, Rh6G, DTDC and DTTC) with the final concentration of $1 \mu \mathrm{M}$. After $30 \mathrm{~min}, 0.1$ $\mathrm{mL}$ of $d \mathrm{BSA}$ solution was added followed by $12 \mathrm{~h}$ of incubation. $d \mathrm{BSA}$ was prepared by chemically treating BSA with $\mathrm{NaBH}_{4}$ accordingly to a previously published procedure. ${ }^{37}$ BSA reduced under this condition would have most of its disulfide bonds converted into thiol groups. The $d$ BSA modified UCF-SERS tags were centrifuged and washed with water twice to remove the excess Raman reporter and $d \mathrm{BSA}$, and then redispersed in $0.5 \mathrm{~mL}$ of water.

MTT Assay. MTT assays were carried out to evaluate the potential cytotoxicity of UCF-SERS tags with U87 glioblastoma-astrocytoma cells, L02 liver cells and MCF-7 breast cancer cells. These three kinds of cells were seeded onto 96-well plates $\left(1 \times 10^{4}\right.$ cells per well), respectively. After cultivation in $5 \% \mathrm{CO}_{2}$ at $37{ }^{\circ} \mathrm{C}$ for $24 \mathrm{~h}$, different concentration of the tags $\left(0,1.6,3.2,6.5,13.0\right.$, and $\left.32.5 \mu \mathrm{g} \mathrm{mL}^{-1}\right)$ were added into the wells and incubated with the cells for $24 \mathrm{~h}$. A stock solution of MTT $\left(20 \mu \mathrm{L}, 5 \mathrm{mg} \mathrm{mL}^{-1}\right)$ was added into each well. After 4 $\mathrm{h}$ incubation at $37^{\circ} \mathrm{C}$, the MTT solution was replaced with $150 \mu \mathrm{L}$ DMSO in each well. The plates were gently shaken for $30 \mathrm{~min}$ at room temperature before measuring the absorbance at the wavelength of 490 $\mathrm{nm}$. The cytotoxicity was expressed as the percentage of cell viability compared to control cells.

Cellular Optical Imaging. UCF- SERS tags were gently mixed with live MCF-7 cells and incubated for $2 \mathrm{~h}$. The culture medium containing free tags were then discarded and the cells were washed with fresh culture medium for three times. UCF imaging of MCF-7 cells was performed on a modified Olympus FV-1000 laser confocal scanning microscope with an external $980 \mathrm{~nm}$ NIR laser. To confirm 

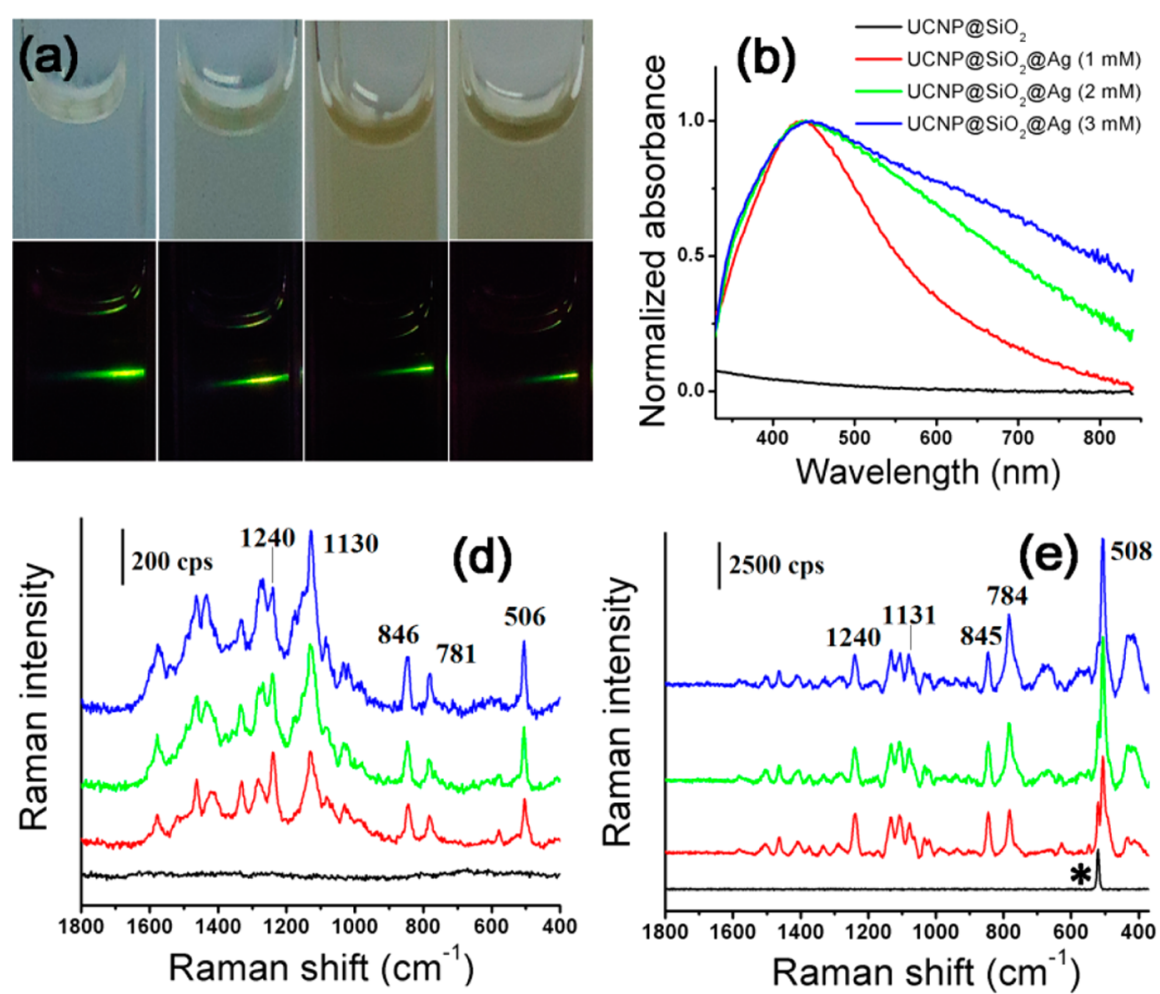

Figure 2. (a) Solution images of UCNP@SiO ${ }_{2}$ and the UCF-SERS tags with 1,2, and $3 \mathrm{mM} \mathrm{Ag}$ (up panel from left to right), and the corresponding fluorescence images upon excitation at $980 \mathrm{~nm}$ (down panel). The samples were diluted 5 times by water before photos were taken. (b, c) Normalized UV/vis/NIR absorption spectra and upconversion fluorescence spectra of UCNP@SiO ${ }_{2}$ and the UCF-SERS tags with 1, 2, and 3 mM Ag, respectively. (d, e) SERS spectra of UCNP@SiO ${ }_{2}$ and the UCF-SERS tags with 1, 2, and $3 \mathrm{mM} \mathrm{Ag}$ recorded with 632.8 and $785 \mathrm{~nm}$ irradiation, respectively. The Raman reporter was DTTC and the peak at $520 \mathrm{~cm}^{-1}$ indicating with asterisk originated from the silicon wafer. (f) Rlationship between dual mode optical intensities and the Ag content of the UCF-SERS tags.

the uptake of the tags by the cells, three-dimensional confocal imaging was taken at different depths along the $z$-axis. SERS spectra of the labeled cells were recorded by a DXR Raman microscope with a $50 \times$ microscope objective. The wavelength of the laser was $632.8 \mathrm{~nm}$ and the power was $2 \mathrm{~mW}$.

In Vivo Optical Imaging. $50 \mu \mathrm{L}$ of the tag solution was injected subcutaneously at the abdomen of an anaesthetized Kunming mouse. The UCF at the injection position irradiated by $980 \mathrm{~nm}$ laser $(200$ $\mathrm{mW}$ ) was captured via a digital single lens reflex camera (Canon, Japan). The subcutaneous SERS spectrum was detected using an Agility Raman spectrometer (BaySpec, USA). The laser power was $200 \mathrm{~mW}$ and the integration time was $1 \mathrm{~s}$. The control spectrum was taken in an area away from the injected site.

\section{RESULTS AND DISCUSSION}

Morphology of UCNP@SiO ${ }_{2} @$ Ag Nanocomposite. The morphology of the prepared NPs was characterized by TEM. As shown in Figure 1a, the $\mathrm{NaYF}_{4}: \mathrm{Yb}, \mathrm{Er}$ UCNP was sphere with an average diameter around $35 \mathrm{~nm}$. The TEM image of UCNP@SiO ${ }_{2}$ NPs with about $10 \mathrm{~nm}$ silica shell was shown in Figure $1 \mathrm{~b}$. The silica coating not only made the UCNPs hydrophilic but also provided a gap with optimal distance between UCNPs and further growing Ag NPs, which was beneficial for UCNPs to achieve the maximum luminescence enhancement. ${ }^{34} \mathrm{Ag}$ NPs were in situ grown on thiol modified $\mathrm{UCNP} @ \mathrm{SiO}_{2} \mathrm{NPs}$ through reduction of $\mathrm{AgNO}_{3}$ by ethanolamine in ethylene glycol. Figure 1c-e showed the morphology of UCNP@SiO $\mathrm{S}_{2} @ \mathrm{Ag}$ nanocomposites prepared with different concentrations of $\mathrm{AgNO}_{3}(1,2$, and $3 \mathrm{mM}$ ). These images revealed that the size and coating density of Ag NPs could be tuned by adjusting $\mathrm{AgNO}_{3}$ concentration in the reaction mixture. When the concentration of $\mathrm{AgNO}_{3}$ was $1 \mathrm{mM}$, more than $90 \%$ of Ag NPs was in the range of 5 to $15 \mathrm{~nm}$, whereas the percentage of Ag NPs lager than $15 \mathrm{~nm}$ continuously grew with the concentration of $\mathrm{AgNO}_{3}$ increasing to $3 \mathrm{mM}$ (Figure if $-\mathrm{h})$.

Optical Properties of $d$ BSA-Capped UCF-SERS Tag. $d$ BSA protected UCF-SERS tags possessed excellent water solubility and the following studies were all performed in aqueous media. The up panel in Figure 2a (from left to right) showed the sample images of $\mathrm{UCNP} @ \mathrm{SiO}_{2}$ and the tags with 1, 2 , and $3 \mathrm{mM} \mathrm{Ag}$, respectively. The color of the solutions turned from colorless to yellow and finally became dark brown with increasing amount of Ag. Upon excitation at $980 \mathrm{~nm}$, apparent fluorescence was observed from these samples, which appearing predominantly green color (Figure 2a, down panel).

To characterize the color variation, UV/vis/NIR absorption spectra of these samples were measured. As illustrated in Figure 2b, UCNP@SiO $\mathrm{S}_{2}$ sample showed no apparent absorption. UCF-SERS tag $(1 \mathrm{mM} \mathrm{Ag})$ sample displayed an absorption peaks around $435 \mathrm{~nm}$, corresponding to the SPR band of single Ag NP. A slight red-shift (440 and $447 \mathrm{~nm}$ ) and broadening of the plasmon resonance band were observed for the tags with 2 and $3 \mathrm{mM} \mathrm{Ag}$, respectively, which was contributed by the growing size of coated $\mathrm{Ag} \mathrm{NPs}$ and the formation of $\mathrm{Ag}$ clusters.

The fluorescence spectra of the four samples displayed two emission bands centered at 542 and $658 \mathrm{~nm}$ under the excitation at $980 \mathrm{~nm}$ (Figure 2c), corresponding to ${ }^{2} \mathrm{H}_{11 / 2} /{ }^{4} \mathrm{~S}_{3 / 2}{ }^{4} \mathrm{II}_{5 / 2}$ and ${ }^{4} \mathrm{~F}_{9 / 2}{ }^{4} \mathrm{I}_{5 / 2}$ transitions of UCNP, respectively. ${ }^{36}$ The coating of $\mathrm{Ag}$ NPs did not change the 
fluorescence spectra profile of UCNPs obviously. However, the emission intensity of the tags was tuned. For both UCF-SERS tags with 1 and $2 \mathrm{mM} \mathrm{Ag}$, the fluorescence of UCNP was enhanced and the enhancement factor was determined to be 1.74 and 1.81, respectively. It was regarded that Ag NPs influenced the emission process of UCNPs mainly in two ways, i.e., the enhanced radiative decay which could increase emission intensity, and metal induced energy transfer which resulted in the fluorescence quenching. As the separation distance between Ag NPs and UCNPs increased, both effects would be weakening. However, the energy transfer rate decreased more rapidly. Therefore, the enhanced radiative decay rate would take a dominant role so that enhanced fluorescence could be obtained. ${ }^{34}$ When the $\mathrm{Ag}$ concentration was $3 \mathrm{mM}$, the fluorescence intensity of the nanocomposites declined to about $83 \%$ intensity of $\mathrm{UCNP} @ \mathrm{SiO}_{2}$ precursor. This was possibly caused by the enhanced inner filter effect resulting from the growing number and size of Ag NPs on UCNP@SiO

Furthermore, the influence of different Ag NP coating conditions on SERS signals of UCF-SERS tags was investigated by using DTTC as the Raman reporter. As shown in Figure 2d, e, no SERS signal could be detected from UCNP@SiO ${ }_{2} \mathrm{NPs}$, whereas characteristic Raman peaks of DTTC around 506, 781, 846,1130 , and $1240 \mathrm{~cm}^{-1}$ were observed for three tags under both $632.8 \mathrm{~nm}$ and $785 \mathrm{~nm}$ laser irradiations. The SERS intensity increased with the growing amount of Ag. This SERS increasing trend was attributed to the following reasons: (1) The size of single Ag NPs played a crucial role for SERS signal enhancement ability. ${ }^{38}$ The Ag NPs in UCF-SERS tags were reaching the optimal size $(30-50 \mathrm{~nm})$ for SERS application upon continuous growing. (2) The growth process made the adjacent Ag NPs very close, thus form a proportion of NP dimers and small clusters. Intense electromagnetic field would be generated at the junctions between NPs (hotspots), which greatly increased the SERS intensity of Raman reporters in the tags. ${ }^{1}$

Figure $2 \mathrm{f}$ showed the connections between dual mode optical intensities and the $\mathrm{Ag}$ content of the UCF-SERS tags. The SERS intensity of $1130 \mathrm{~cm}^{-1}$ peak (632.8 nm irradiation) continuously increased (the trend was similar with $785 \mathrm{~nm}$ irradiation), while the UCF underwent a growing-declining process with the $\mathrm{Ag}$ content increasing from 1 to $3 \mathrm{mM}$. To make a compromise of these two factors, the UCF-SERS tags $(2 \mathrm{mM} \mathrm{Ag})$ were in the following experiments.

To render multiplex labeling ability of the tags, five kinds of dye molecules (MG, CV, Rh6G, DTDC and DTTC) were tested as Raman reporters by using UCNP@SiO $\mathrm{S}_{2} @ \mathrm{Ag}(2 \mathrm{mM})$ nanocomposites as SERS substrates. As illustrated in Figure S1a in the Supporting Information, the characteristic Raman peaks could be observed for all five dyes by using a $632.8 \mathrm{~nm}$ laser source, among which $\mathrm{CV}$ and $\mathrm{MG}$ labeled tags showed extremely high intensities. By contrast, when $785 \mathrm{~nm}$ NIR laser was applied, only DTTC showed strong SERS signal, and those of the other four reporters were too weak to be detected (see Figure S1b in the Supporting Information). Previous studies demonstrated that the absorption feature of reporter molecules dramatically affected the Raman signal enhancing ability. When the absorption matched the excitation-laser's wavelength, surface enhanced resonant Raman scattering (SERRS) occurred, and the enhancement factor might be further enhanced 100 times. ${ }^{39}$ In our experiment, the maximum absorption of MG $(615 \mathrm{~nm})$ and CV $(589 \mathrm{~nm})$ had a good match with $632.8 \mathrm{~nm}$ laser while that of DTTC $(764 \mathrm{~nm})$ was close to $785 \mathrm{~nm}$ (see Figure S2 in the Supporting Information). Therefore, these Raman reporters showed strong characteristic signals at the corresponding irradiation conditions. The above results implied that UCF-SERS tags with multiple SERS signatures for cell labeling (with $632.8 \mathrm{~nm}$ irradiation) could be obtained, whereas only the tag with DTTC as the Raman reporter met the requirement for both cell and in vivo imaging (with $785 \mathrm{~nm}$ irradiation). Therefore, DTTC labeled UCFSERS tags were applied in the following study and the signal stability of both signals of the tags were satisfactory (see Figure S3 in the Supporting Information).

We further investigated the influence of $d \mathrm{BSA}$ modification on the sensing capability of the tags. We measured the upconversion fluorescence and SERS signals (irradiated with 632.8 nm laser) of DTTC labeled UCNP@SiO $@$ @Ag (2 mM) nanocomposites before and after attaching $d \mathrm{BSA}$. As shown in Figure $\mathrm{S} 4$ in the Supporting Information, $d B S A$ molecule almost did not affect both optical features of the tags. The tags were monodispersive and stable in water and $\mathrm{pH} 7.4$ phosphate buffered saline (see Figure S5 in the Supporting Information), which were ready for biological labeling investigations.

Cytotoxicity Study and Cell Imaging. The cytotoxicity of the UCF-SERS tags was estimated by MTT assays on U87 glioblastoma-astrocytoma cells, L02 normal liver cells and MCF-7 breast cancer cells. It had been reported that UCNP@ $\mathrm{SiO}_{2} @ \mathrm{Ag}$ nanocomposites were cytotoxic, which might be arisen from the ethanolamine-capped Ag NPs. ${ }^{34}$ To overcome this problem, the biocompatible $d \mathrm{BSA}$ was used as the capping agent of the tags by taking the place of ethanolamine through $\mathrm{Ag}-\mathrm{S}$ bonds between $\mathrm{Ag} \mathrm{NPs}$ and multiple thiol groups in $d \mathrm{BSA}$. The MTT results indicated that there were no significant decreases in cell viability even after incubation at the highest dose of $d$ BSA protected UCF-SERS tags $\left(32.5 \mu \mathrm{g} \mathrm{mL}^{-1}\right)$ (Figure 3).

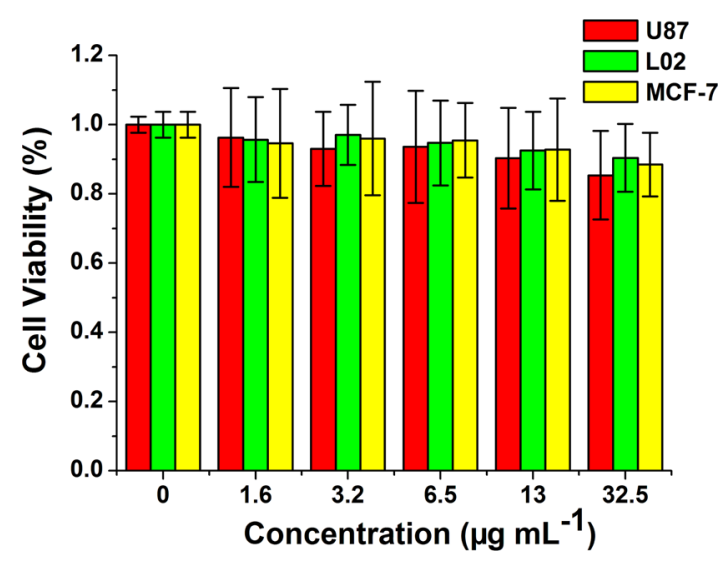

Figure 3. Cytotoxicity assays of the UCF-SERS tags in U87, L02, and MCF-7 cells.

To monitor the intracellular uptake and dual mode optical labeling property of UCF-SERS tags, MCF-7 cells were incubated with the tags for $2 \mathrm{~h}$. After washing the free NPs in culture medium, the cells were imaged in bright field and fluorescence field respectively by an Olympus confocal microscope equipped with a $980 \mathrm{~nm}$ NIR laser. Obvious green and red UCF were simultaneously observed at the cancer cells. Additionally, taking advantage of the "optical sectioning" function of laser confocal fluorescence microscope, fluorescence images were acquired at different confocal depth (along z- axis) 
(a)

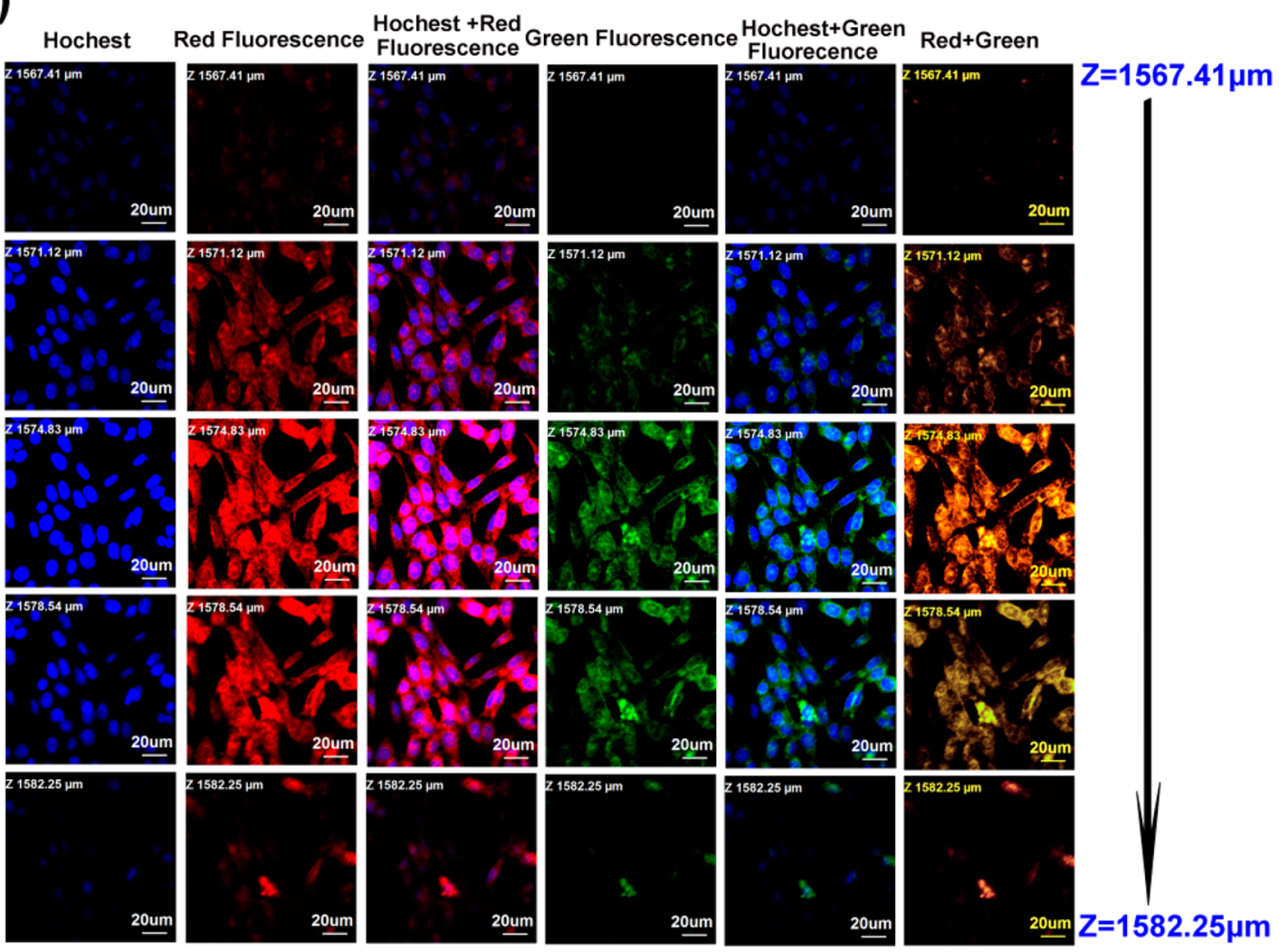

(b)

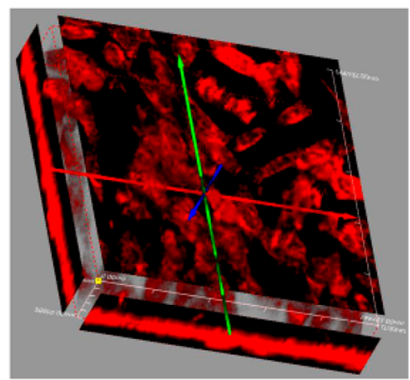

(c)

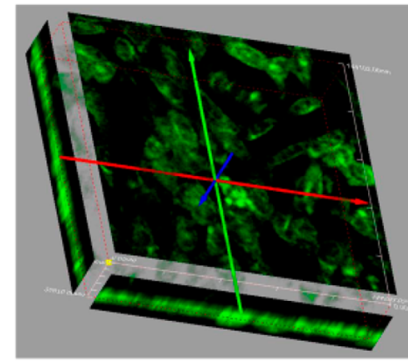

Figure 4. Cell uptake of $d$ BSA-capped UCF-SERS tag in MCF-7 cells. (a) Series images of the xy planes (z-slice) of tag incubated MCF-7 cells at the continuative $z$-axis (from $1567.41 \mu \mathrm{m}$ to $1582.25 \mu \mathrm{m}$ ). Hoechst 33342 was used for cell nuclei staining (blue fluorescence). Red and green fluorescence in the MCF-7 cells were all originated from UCNPs. Three-dimensional reconstruction of (b) the red fluorescence and (c) green fluorescence confocal images along the $z$-axis. Arrows indicate the spatial orientation of objects with respect to the $x, y$, and $z$-axis (red, green, and blue, respectively).
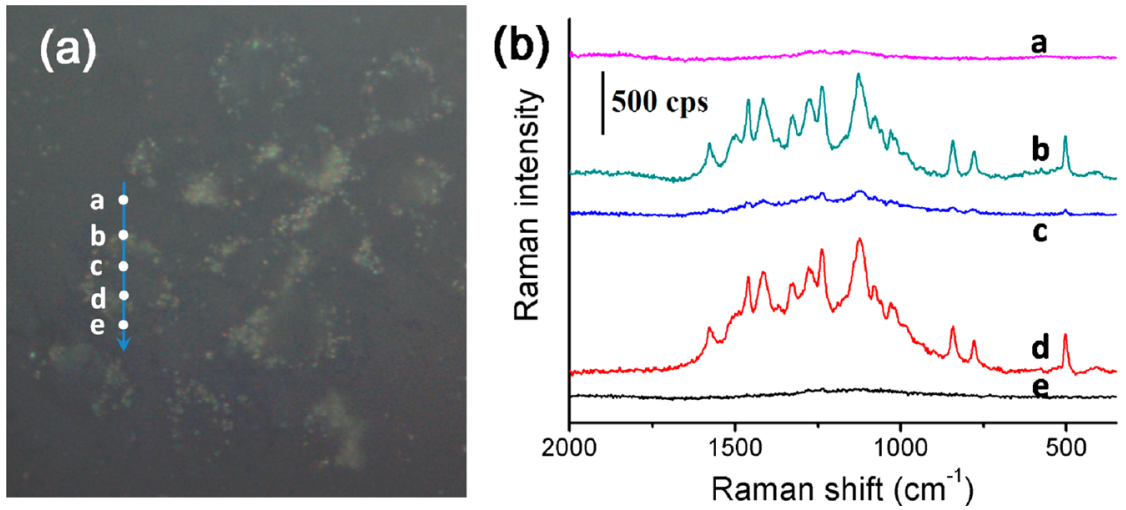

Figure 5. (a) Dark-field images of MCF-7 cells after incubating UCF-SERS tags for $2 \mathrm{~h}$. (b) Corresponding Raman spectra at five different laser spots (a-e) across one tag-labeled MCF-7 cell. 


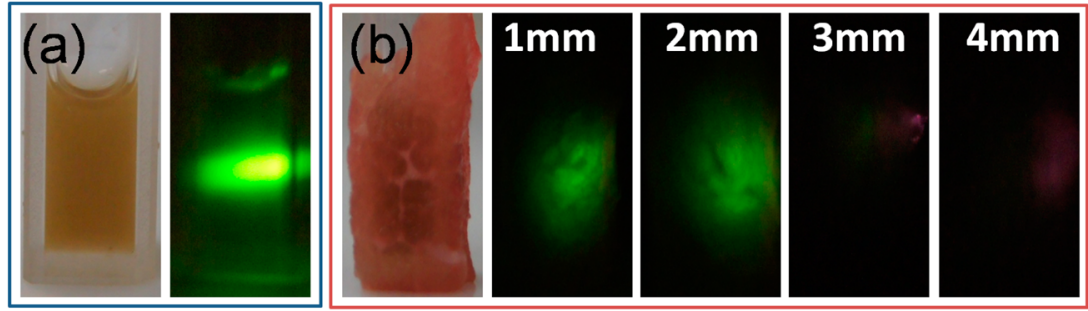

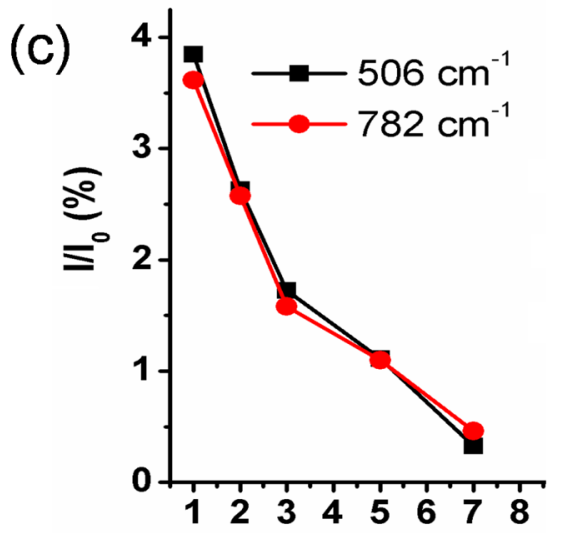

Tissue thickness $(\mathrm{mm})$
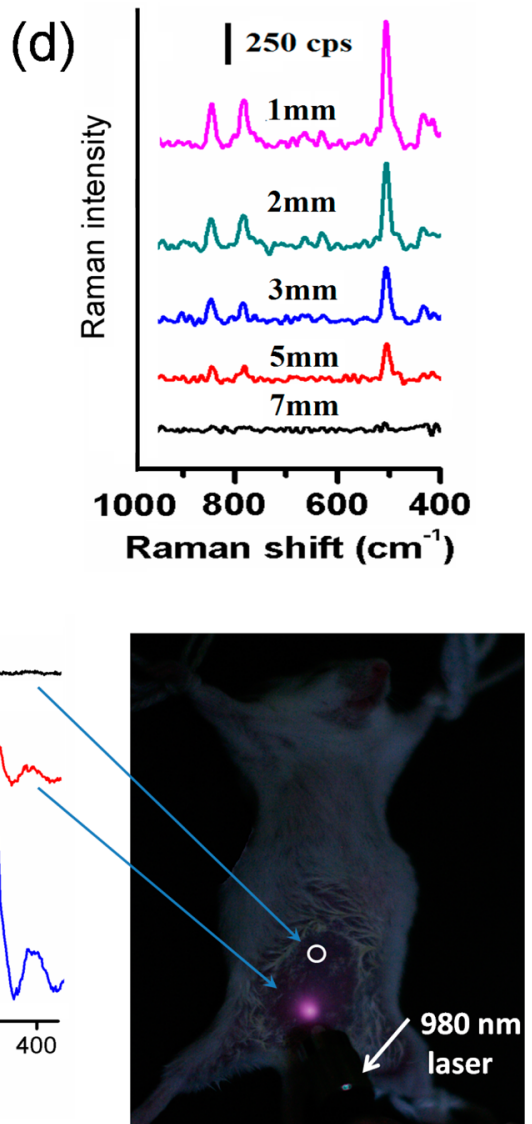

Figure 6. (a) Normal and fluorescence images of $100 \mu \mathrm{L}$ UCF-SERS tag $\left(0.65 \mathrm{mg} \mathrm{mL}^{-1}\right)$ solution in cuvette. (b) The image of the pork tissue slices wrapped cuvette and the fluorescence images captured from the samples covered with tissue of different thickness with the excitation of $980 \mathrm{~nm}$ laser $(1.0 \mathrm{~W})$. (c) The relationship between SERS intensity of UCF-SERS tag solution and the thickness of the covered pork tissue. I denoted the intensity at peak $1130 \mathrm{~cm}^{-1}$ of the corresponding measurement with pork tissue and $\mathrm{I}_{0}$ denoted the intensity of this peak without any coverage. (d) SERS spectra of the tags as a function of pork tissue thickness. (e) Upconversion fluorescence photograph of subcutaneously injected with $50 \mu \mathrm{L}$ of UCF-SERS tag solution $\left(0.65 \mathrm{mg} \mathrm{mL}^{-1}\right)$ in a Kunming rat under $980 \mathrm{~nm}$ excitation $\left(0.5 \mathrm{~W} / \mathrm{cm}^{2}\right)$. SERS spectra from the tag injected site and the blank skin with the $785 \mathrm{~nm}$ irradiation $(200 \mathrm{~mW}$, acquisition time $1 \mathrm{~s})$.

(Figure 4a). Obviously the tags entered the cells, as evidenced by fluorescence signals appeared along the $\mathrm{Z}$ axis. Meanwhile, the slice fluorescence images were reconstructed in threedimensions (Figure 4b, c). All the results clearly indicated that a large amount of the tags were spread in cytoplasmic regions of the cells and the green and red fluorescence were co-localized inside the cells. By contrast, the control cells incubated without the tags showed no fluorescence under similar imaging conditions (see Figure S6 in the Supporting Information).

Furthermore, we investigated the intracellular SERS performance of the dual-mode imaging tag by Raman microscopy with the excitation wavelength of $632.8 \mathrm{~nm}$. Figure 5 showed a darkfield image of the tag-labeled MCF-7 cells, and SERS spectra at five different laser spots across one cell. Obviously, strong characteristic Raman peaks of DTTC were recorded from
UCF-SERS tags accumulated in cytoplasma. The SERS signals at the nucleus and the glass slide were nearly undetected.

Dual Mode Imaging Investigation in Vivo. To demonstrate the effectiveness of UCF-SERS tags for imaging of deep tissue, we wrapped a cuvette containing $100 \mu \mathrm{L}$ of the tags with slices of pork tissue of different thickness, and then recorded the fluorescence and SERS signals. Figure 6a showed the images of the sample and fluorescence obtained with the excitation of $980 \mathrm{~nm}$ laser. Strong green upconverted emission could be monitored from the cuvette. Figure $6 \mathrm{~b}$ illustrated the images of the pork tissue wrapped cuvette and fluorescence captured from the samples covered with tissue of different thickness. At the imaging depth of 1 and $2 \mathrm{~mm}$, clear fluorescence could be observed, illustrating their favorable capability for superficial layer in vivo fluorescent imaging. As the thickness of pork tissue increased, the fluorescence declined 
sharply $(3 \mathrm{~mm})$ and became nearly undetected when the thickness reached $4 \mathrm{~mm}$.

Subsequently, the SERS signals were measured from the tissue wrapped cuvett via Agility Raman spectrometer with a NIR excitation laser $(785 \mathrm{~nm})$. As shown in Figure $6 \mathrm{c}$, the SERS intensity of UCF-SERS tag solution sharply decreased with the coverage of pork tissue. The intensity of both Raman peaks at 506 and $782 \mathrm{~cm}^{-1}$ dropped to about $4 \%$ of their initial values after being coated with $1 \mathrm{~mm}$ tissue. With the depth of tissue increasing, the SERS signals underwent a continuous decrease. However, it could be seen from Figure $6 \mathrm{~d}$ that despite there being a great loss of the SERS intensity compared with the sole tag solution, detectable SERS signal could still be obtained even from the cuvette wrapped with pork tissue coating as thick as $7 \mathrm{~mm}$. These results indicated that the SERS signals generated from UCF-SERS tags could be detected from deep tissues and were sufficient for in vivo detection.

Finally, the in vivo dual mode imaging performance of the UCF-SERS tags was evaluated in a mouse. A portion of $50 \mu \mathrm{L}$ of the tag solution was injected subcutaneously at the abdomen of an anaesthetized Kunming mouse. As indicated in Figure 6e, the UCF could be seen at the injection positions with naked eyes upon the irradiation of $980 \mathrm{~nm}$ laser $(200 \mathrm{~mW})$. SERS measurement was also successfully carried out. No Raman signal could be observed from the animal skin. By contrast, the spectral signatures of F-SERS tags could be clearly distinguished at the injection location through the mouse skin, despite the intensity decreased to about a quarter of that of pure tag solution. These results proved the high sensitivity of our UCF-SERS tag and its potential to be used for future UCF and SERS dual mode imaging studies in animal models.

\section{CONCLUSION}

In summary, we proposed the first UCF-SERS dual mode tag for living cell and in vivo bioimaging. Satisfactory fluorescence sensitivity was achieved because the fluorescence of UCNP was enhanced by the attached Ag NPs serving as SERS substrates. The selection of DTTC Raman reporter generated strong SERS signal, which was sufficient for both cell and deep tissue detection. Taking advantages of these superior optical properties, in vivo imaging of UCF-SERS tags were successfully demonstrated on a living mouse by using $980 \mathrm{~nm}$ and $785 \mathrm{~nm}$ NIR irradiation.

Although this work described a proof-of-concept study of UCF-SERS dual mode tags, such tags held great potential for medical diagnostics and therapy with further improvement. First, although the excitation wavelength of UCNPs in this work was $980 \mathrm{~nm}$, the emission was located in the visible region. We believed that there was still plenty of room to improve in vivo imaging sensitivity by the application of novel type of NIR in-NIR out UCNP. ${ }^{40}$ Second, after screening more Raman reporters sensitive to $785 \mathrm{~nm}$ irradiation, in vivo multiplex labeling and detection using SERS techniques could be realized. Third, the biocompatibility of our tag can be further improved by taking place of silver NPs by gold nanoshells, or further coating silica shells on the surface of Ag NPs. Fourth, by proper surface modification with tumor-targeting molecules, the dual mode tags were promising to be applied for pre-clinical tumor diagnosis.

\section{ASSOCIATED CONTENT}

\section{Supporting Information}

SERS signals of UCF-SERS tags with different Raman reporters and different irradiation laser sources. UCF and SERS signal stability of the tags. The influence of $d \mathrm{BSA}$ attachment on the optical features of the tags. The hydrodynamic diameter results of the samples. LCSM image of control MCF-7 cells. This material is available free of charge via the Internet at http:// pubs.acs.org.

\section{AUTHOR INFORMATION}

\section{Corresponding Authors}

* E-mail: yqwang@yic.ac.cn. Tel.: +86 535 2109130. Fax: +86 5352109000.

*E-mail: 1xchen@yic.ac.cn.

\section{Author Contributions}

${ }^{\dagger}$ Authors X.N. and H.C. contributed equally to this work.

\section{Notes}

The authors declare no competing financial interest.

\section{ACKNOWLEDGMENTS}

We acknowledge financial support from the National Natural Science Foundation of China (81102415, 21305157, 21275158, 81371684 and 81000666), the Natural Science Foundation of Shandong Province of China (ZR2010BQ012), the Science and Technology Development Plan of Yantai (2011071), and the One Hundred Person Project of the Chinese Academy of Sciences.

\section{REFERENCES}

(1) Wang, Y.; Yan, B.; Chen, L. SERS Tags: Novel Optical Nanoprobes for Bioanalysis. Chem. Rev. 2013, 113, 1391-1428.

(2) Li, J.; Chen, L.; Lou, T.; Wang, Y. Highly Sensitive SERS Detection of $\mathrm{As}^{3+}$ Ions in Aqueous Media Using Glutathione Functionalized Silver Nanoparticles. ACS Appl. Mater. Interfaces 2011, 3, 3936-3941.

(3) Wang, X.; Chen, L.; Fu, X.; Ding, Y. Highly Sensitive SurfaceEnhanced Raman Scattering Sensing of Heparin Based on AntiAggregation of Functionalized Silver Nanoparticles. ACS Appl. Mater. Interfaces 2013, 5, 11059-11065.

(4) Lou, T.; Wang, Y.; Li, J.; Peng, H.; Xiong, H.; Chen, L. Rapid Detection of Melamine with 4-Mercaptopyridine-Modified Gold Nanoparticles by Surface-Enhanced Raman Scattering. Anal. Bioanal.Chem. 2011, 401, 333-338.

(5) Wang, Y.; Chen, L.; Liu, P. Biocompatible Triplex Ag@SiO $\mathrm{mTiO}_{2}$ Core-Shell Nanoparticles for Simultaneous Fluorescence-SERS Bimodal Imaging and Drug Delivery. Chem.Eur. J. 2012, 18, 59355943.

(6) Lin, D.; Qin, T.; Wang, Y.; Sun, X.; Chen, L. Graphene Oxide Wrapped SERS Tags: Multifunctional Platforms toward Optical Labeling, Photothermal Ablation of Bacteria, and the Monitoring of Killing Effect. ACS Appl. Mater. Interfaces 2014, 6, 1320-1329.

(7) Kustner, B.; Gellner, M.; Schutz, M.; Schoppler, F.; Marx, A.; Strobel, P.; Adam, P.; Schmuck, C.; Schlucker, S. SERS Labels for Red Laser Excitation: Silica-Encapsulated SAMs on Tunable Gold/Silver Nanoshells. Angew.Chem. Int. Ed. 2009, 48, 1950-1953.

(8) Kang, H.; Jeong, S.; Park, Y.; Yim, J.; Jun, B.-H.; Kyeong, S.; Yang, J.-K.; Kim, G.; Hong, S.; Lee, L. P.; Kim, J.-H.; Lee, H.-Y.; Jeong, D. H.; Lee, Y.-S. Near-Infrared SERS Nanoprobes with Plasmonic Au/ Ag Hollow-Shell Assemblies for in Vivo Multiplex Detection. Adv. Funct. Mater. 2013, 23, 3719-3727.

(9) Wang, Z.; Zong, S.; Yang, J.; Li, J.; Cui, Y. Dual-Mode Probe Based on Mesoporous Silica Coated Gold Nanorods for Targeting Cancer Cells. Biosens. Bioelectron. 2011, 26, 2883-2889. 
(10) Lee, S.; Chon, H.; Yoon, S. Y.; Lee, E. K.; Chang, S. I.; Lim, D. W.; Choo, J. Fabrication of SERS-Fluorescence Dual Modal Nanoprobes and Application to Multiplex Cancer Cell Imaging. Nanoscale 2012, 4, 124-129.

(11) Wang, Z.; Zong, S.; Chen, H.; Wu, H.; Cui, Y. Silica Coated Gold Nanoaggregates Prepared by Reverse Microemulsion Method: Dual Mode Probes for Multiplex Immunoassay Using SERS and Fluorescence. Talanta 2011, 86, 170-177.

(12) Xiao, M.; Nyagilo, J.; Arora, V.; Kulkarni, P.; Xu, D.; Sun, X.; Dave, D. P. Gold Nanotags for Combined Multi-Colored Raman Spectroscopy and X-Ray Computed Tomography. Nanotechnology 2010, 21, 035101.

(13) Yigit, M. V.; Zhu, L. Y.; Ifediba, M. A.; Zhang, Y.; Carr, K.; Moore, A.; Medarova, Z. Noninvasive MRI-SERS Imaging in Living Mice Using an Innately Bimodal Nanomaterial. ACS Nano 2011, 5, $1056-1066$.

(14) Kircher, M. F.; de la Zerda, A.; Jokerst, J. V.; Zavaleta, C. L.; Kempen, P. J.; Mittra, E.; Pitter, K.; Huang, R. M.; Campos, C.; Habte, F.; Sinclair, R.; Brennan, C. W.; Mellinghoff, I. K.; Holland, E. C.; Gambhir, S. S. A Brain Tumor Molecular Imaging Strategy Using a New Triple-Modality Mri-Photoacoustic-Raman Nanoparticle. Nat. Med. 2012, 18, 829-834.

(15) Qian, J.; Jiang, L.; Cai, F. H.; Wang, D.; He, S. L. FluorescenceSurface Enhanced Raman Scattering Co-Functionalized Gold Nanorods as near-Infrared Probes for Purely Optical in Vivo Imaging. Biomaterials 2011, 32, 1601-1610.

(16) Zhang, Y.; Qian, J.; Wang, D.; Wang, Y.; He, S. Multifunctional Gold Nanorods with Ultrahigh Stability and Tunability for in Vivo Fluorescence Imaging, SERS Detection, and Photodynamic Therapy. Angew. Chem., Int. Ed. 2013, 52, 1148-1151.

(17) Yu, K. N.; Lee, S. M.; Han, J. Y.; Park, H.; Woo, M. A.; Noh, M. S.; Hwang, S. K.; Kwon, J. T.; Jin, H.; Kim, Y. K.; Hergenrother, P. J.; Jeong, D. H.; Lee, Y. S.; Cho, M. H. Multiplex Targeting, Tracking, and Imaging of Apoptosis by Fluorescent Surface Enhanced Raman Spectroscopic Dots. Bioconjugate Chem. 2007, 18, 1155-1162.

(18) Woo, M. A.; Lee, S. M.; Kim, G.; Baek, J.; Noh, M. S.; Kim, J. E.; Park, S. J.; Minai-Tehrani, A.; Park, S. C.; Seo, Y. T.; Kim, Y. K.; Lee, Y. S.; Jeong, D. H.; Cho, M. H. Multiplex Immunoassay Using Fluorescent-Surface Enhanced Raman Spectroscopic Dots for the Detection of Bronchioalveolar Stem Cells in Murine Lung. Anal. Chem. 2009, 81, 1008-1015.

(19) Kim, K.; Lee, Y. M.; Lee, H. B.; Shin, K. S. Silver Salts of Aromatic Thiols Applicable as Core Materials of Molecular Sensors Operating Via SERS and Fluorescence. Biosens. Bioelectron. 2009, 24, $3615-3621$.

(20) Kim, K.; Lee, H. B.; Lee, Y. M.; Shin, K. S. Rhodamine B Isothiocyanate-Modified Ag Nanoaggregates on Dielectric Beads: A Novel Surface-Enhanced Raman Scattering and Fluorescent Imaging Material. Biosens. Bioelectron. 2009, 24, 1864-1869.

(21) Wang, Z.; Wu, H.; Wang, C.; Xu, S.; Cui, Y. Gold Aggregatesand Quantum Dots- Embedded Nanospheres: Switchable Dual-Mode Image Probes for Living Cells. J. Mater. Chem. 2011, 21, 4307.

(22) Wang, Z.; Zong, S.; Li, W.; Wang, C.; Xu, S.; Chen, H.; Cui, Y. SERS-Fluorescence Joint Spectral Encoding Using Organic-Metal-Qd Hybrid Nanoparticles with a Huge Encoding Capacity for HighThroughput Biodetection: Putting Theory into Practice. J. Am. Chem. Soc. 2012, 134, 2993-3000.

(23) Wang, Z. Y.; Zong, S. F.; Li, W.; Wang, C. L.; Xu, S. H.; Chen, H.; Cui, Y. P. SERS-Fluorescence Joint Spectral Encoding Using Organic-Metal-QD Hybrid Nanoparticles with a Huge Encoding Capacity for High-Throughput Biodetection: Putting Theory into Practice. J. Am. Chem. Soc. 2012, 134, 2993-3000.

(24) Wang, F.; Liu, X. Recent Advances in the Chemistry of Lanthanide-Doped Upconversion Nanocrystals. Chem. Soc. Rev. 2009, 38, 976-989.

(25) Gu, Z.; Yan, L.; Tian, G.; Li, S.; Chai, Z.; Zhao, Y. Recent Advances in Design and Fabrication of Upconversion Nanoparticles and Their Safe Theranostic Applications. Adv. Mater. 2013, 25, 37583779.
(26) Gorris, H. H.; Wolfbeis, O. S. Photon-Upconverting Nanoparticles for Optical Encoding and Multiplexing of Cells, Biomolecules, and Microspheres. Angew. Chem., Int. Ed. 2013, 52, 3584-3600.

(27) Cheng, L.; Wang, C.; Liu, Z. Upconversion Nanoparticles and Their Composite Nanostructures for Biomedical Imaging and Cancer Therapy. Nanoscale 2013, 5, 23-37.

(28) Cui, S.; Chen, H.; Zhu, H.; Tian, J.; Chi, X.; Qian, Z.; Achilefu, S.; Gu, Y. Amphiphilic Chitosan Modified Upconversion Nanoparticles for in Vivo Photodynamic Therapy Induced by near-Infrared Light. J. Mater. Chem. 2012, 22, 4861.

(29) Cui, S. S.; Yin, D. Y.; Chen, Y. Q.; Di, Y. F.; Chen, H. Y.; Ma, Y. X.; Achilefu, S.; Gu, Y. Q. In Vivo Targeted Deep-Tissue Photodynamic Therapy Based on near-Infrared Light Triggered Upconversion Nanoconstruct. ACS Nano 2013, 7, 676-688.

(30) Cheng, L.; Yang, K.; Li, Y.; Chen, J.; Wang, C.; Shao, M.; Lee, S. T.; Liu, Z. Facile Preparation of Multifunctional Upconversion Nanoprobes for Multimodal Imaging and Dual-Targeted Photothermal Therapy. Angew. Chem. Int. Ed. 2011, 50, 7385-7390.

(31) Liu, J.; Bu, W.; Pan, L.; Shi, J. Nir-Triggered Anticancer Drug Delivery by Upconverting Nanoparticles with Integrated AzobenzeneModified Mesoporous Silica. Angew. Chem., Int. Ed. 2013, 52, 43754379.

(32) Xiao, Q.; Zheng, X.; Bu, W.; Ge, W.; Zhang, S.; Chen, F.; Xing, H.; Ren, Q.; Fan, W.; Zhao, K.; Hua, Y.; Shi, J. A Core/Satellite Multifunctional Nanotheranostic for In Vivo Imaging and Tumor Eradication by Radiation/Photothermal Synergistic Therapy. J. Am. Chem. Soc. 2013, 135, 13041-13048.

(33) Kannan, P.; Abdul Rahim, F.; Chen, R.; Teng, X.; Huang, L.; Sun, H.; Kim, D. H. Au Nanorod Decoration on $\mathrm{NaYF}_{4}: \mathrm{Yb} / \mathrm{Tm}$ Nanoparticles for Enhanced Emission and Wavelength-Dependent Biomolecular Sensing. ACS Appl. Mater. Interfaces 2013, 5, 35083513.

(34) Yuan, P.; Lee, Y. H.; Gnanasammandhan, M. K.; Guan, Z.; Zhang, Y.; Xu, Q. H. Plasmon Enhanced Upconversion Luminescence of $\mathrm{NaYF}_{4}: \mathrm{Yb}, \mathrm{Er} @ \mathrm{SiO}_{2} @ \mathrm{Ag}$ Core-Shell Nanocomposites for Cell Imaging. Nanoscale 2012, 4, 5132-5137.

(35) Kannan, P.; Rahim, F. A.; Teng, X.; Chen, R.; Sun, H. D.; Huang, L.; Kim, D. H. Enhanced Emission of $\mathrm{NaYF}_{4}: \mathrm{Yb}, \mathrm{Er} / \mathrm{Tm}$ Nanoparticles by Selective Growth of Au and Ag Nanoshells. RSC Adv. 2013, 3, 7718-7721.

(36) Li, Z.; Zhang, Y. An Efficient and User-Friendly Method for the Synthesis of Hexagonal-Phase $\mathrm{NaYF}_{4}: \mathrm{Yb}, \mathrm{Er} / \mathrm{Tm}$ Nanocrystals with Controllable Shape and Upconversion Fluorescence. Nanotechnology 2008, 19, 345606.

(37) Xie, J. P.; Zhang, Q. B.; Lee, J. Y.; Wang, D. I. C. The Synthesis of SERS-Active Gold Nanoflower Tags for in Vivo Applications. ACS Nano 2008, 2, 2473-2480.

(38) Seney, C. S.; Gutzman, B. M.; Goddard, R. H. Correlation of Size and Surface-Enhanced Raman Scattering Activity of Optical and Spectroscopic Properties for Silver Nanoparticles. J. Phys. Chem. C 2009, 113, 74-80.

(39) McNay, G.; Eustace, D.; Smith, W. E.; Faulds, K.; Graham, D. Surface-Enhanced Raman Scattering (SERS) and Surface-Enhanced Resonance Raman Scattering (SERRS): A Review of Applications. Appl. Spectrosc. 2011, 65, 825-837.

(40) Chen, G. Y.; Shen, J.; Ohulchanskyy, T. Y.; Patel, N. J.; Kutikov, A.; Li, Z. P.; Song, J.; Pandey, R. K.; Agren, H.; Prasad, P. N.; Han, G. $\left(\alpha-\mathrm{NaYF}_{4}: \mathrm{Tm}^{3+}\right) / \mathrm{CaF}_{2}$ Core/Shell Nanoparticles with Efficient nearInfrared to near-Infrared Upconversion for High-Contrast Deep Tissue Bioimaging. ACS Nano 2012, 6, 8280-8287. 